\title{
Migración y escuela: Percepciones del personal docente de Educación General Básica sobre niñez migrante
}

\author{
Migration and School: Perceptions of Primary School Teachers About Migrant Children
}

\section{Migração e escola: percepções do professor primário sobre crianças migrantes}

Carlos Muñoz-Labraña

Universidad de Concepción Concepción, Chile carlosem@udec.cl

iD http://orcid.org/0000-0003-2044-1965

Luis Ajagán-Lester Universidad de Concepción Concepción, Chile luiajagan@udec.cl

iD http://orcid.org/0000-0002-8694-0524

Rosendo Martínez-Rodríguez

Universidad de Valladolid

Segovia, España

rosendo.martinez@uva.es

D http://orcid.org/0000-0003-3816-0432

Bastián Torres-Durán Universidad de Concepción

Concepción, Chile

bastiantorres@udec.cl

iD http://orcid.org/0000-0002-6853-3802

Carlos Muñoz-Grandón Universidad Católica de Temuco

Temuco, Chile

carlos.munoz.grandon@gmail.com https://orcid.org/0000-0003-3747-6929

Karen Gutiérrez-Cortés Universidad de Concepción

Concepción, Chile karengc9@gmail.com. iD https://orcid.org/0000-0002-5636-1409

Recibido • Received • Recebido: 29 / 05 / 2019

Corregido • Revised $\cdot$ Revisado: $29 / 10 / 2020$

Aceptado $\cdot$ Accepted $\cdot$ Aprovado: $03 / 12$ / 2020 
http://doi.org/10.15359/ree.25-1.14

http://www.una.ac.cr/educare

educare@una.ac.cr

Resumen: La investigación ha tenido como objetivo analizar las percepciones que tiene el profesorado de primaria sobre la niñez migrante. El estudio, efectuado en cuatro establecimientos municipales del Gran Concepción, Chile, utilizó como técnica de recogida de la información una entrevista en profundidad, y en él participaron 20 docentes de ambos sexos que se desempeñan en establecimientos educacionales que han recibido niñez migrante en los últimos dos años. Los resultados indican que las personas migrantes han pasado a ser una preocupación en la escuela y un fenómeno que llegó para quedarse, que en ocasiones divide al profesorado y para el cual considera no estar preparado, dado que la niñez migrante complejiza su trabajo en el aula. Perciben la migración como un proceso más próximo a la realidad de otros países y difícil de comprender, sobre todo para el personal docente más antiguo. No obstante, la investigación demuestra que este valora positivamente el aumento en la matrícula, la posibilidad de hablar de la diversidad en el aula, y de justipreciar la realidad del país y del continente. Valora la importancia que posee el lenguaje para comunicarse y justipreciar la importancia del debido respeto que se debe dispensar al profesorado.

Palabras claves: Inmigración; migrante; profesorado de primaria; percepción.

Abstract: The research aimed to analyze the perceptions that primary school teachers have of migrant children. The study was conducted in four public schools in Concepción, Chile. An in-depth interview as a data collection technique was used and involved 20 teachers of both sexes who work in educational establishments that have received migrant children in the last two years. The results indicate that migrants have become a concern in the school and a phenomenon that is here to stay. It is a phenomenon that divides the teaching staff at times, and for which they consider themselves unprepared since migrant children make teaching work in the classroom more complex. They perceive migration as a process closer to other countries' reality and difficult to understand, especially for the long-serving staff. However, the research shows that teachers positively value the increase in enrollment, as well as the possibility of talking about diversity in the classroom and thinking about the country and the continent's reality. They also value the importance of language to communicate and due respect for teachers.

Keywords: Immigration, migrant, primary teacher, perception.

Resumo: O objetivo da pesquisa foi analisar as percepções de professores do ensino fundamental sobre crianças migrantes. $O$ estudo realizado em quatro cidades municipais de Gran Concepción, Chile, tornou-se uma técnica em que as informações foram obtidas em uma entrevista em profundidade e participação. 20 professores de ambos os sexos que trabalham como educadores que receberam crianças migrantes nos últimos dois anos. Os resultados indicam que os migrantes passaram por uma preocupação na Escola e um fenômeno que veio para ficar, que os pais estão divididos nos professores e para os quais não estão preparados, dado que as crianças migrantes se concentraram em seu trabalho em a aula. Perceber a migração como um processo mais próximo da realidade de outros países e de difícil compreensão, especialmente os professores mais antigos. No entanto, a pesquisa mostra que o professor valoriza positivamente o aumento de matrículas, a possibilidade de falar sobre a diversidade em sala de aula e a possibilidade de avaliar a realidade do país e do continente. Avaliar a importância da linguagem para comunicar e justificar a importância do respeito que deve ser dado aos professores.

Palavras-chave: Imigração; migrante; professor elementar; percepção. 


\section{Introducción}

El encuentro entre culturas distintas pertenece más bien a las regularidades de la historia que a los hechos fortuitos o a irregularidades de esta. Históricamente estos encuentros han sido forzados por razones religiosas, políticas, sociales y económicas. Existe evidencia de que estos movimientos son el resultado de revueltas, guerras civiles, guerras religiosas impulsadas por el fanatismo y genocidios, por catástrofes naturales o por el natural deseo de mejorar las condiciones de vida (Ajagan Lester, 2016).

A diferencia de las ocurridas en el pasado, las actuales migraciones masivas poseen al menos tres características fundamentales. Primero, involucran a millones de seres humanos. Segundo, son realizadas rápidamente, en pocos días, y no -como en épocas pretéritas-por medio de lentas movilizaciones. Y tercero, se trata de un fenómeno de dimensiones planetarias.

En Chile, el fenómeno de la migración ha estado presente desde la llegada misma de los primeros conquistadores. Al punto que la existencia de colonias extranjeras a lo largo y ancho de su territorio ha sido parte de su normalidad. A pesar de esta vasta experiencia, la migración en el país ha adquirido actualmente una particularidad sin precedentes, dado que involucra esta vez a miles de personas sudamericanas y del Caribe, que hasta ahora no habían querido o necesitado avecindarse en nuestro territorio.

Así, quienes en el pasado considerábamos pueblos vecinos lejanos -o exóticos, como en el caso de los haitianos- están hoy entre nuestra gente. Lo que nos obliga a responder desde nuestro propio espacio existencial, el del endogrupo, algunas preguntas claves acerca de: ¿Cómo les estamos recibiendo, comprendiendo y acogiendo? ¿Tenemos alguna institucionalidad que nos permita mejorar el cómo lo estamos haciendo? ¿Cómo conversar con ellos y ellas? ¿Con la ayuda de qué conceptos y de qué puntos de orientación?, ¿Debemos asimilarles?, ¿Debemos rechazarles y excluirles de nuestra sociedad o debemos coexistir en conjuntos sin mayor contacto? Preguntas que implican evidentes desafíos para enfrentar las migraciones masivas.

La escuela, desde su origen y por definición ha estado asociada al Estado nacional homogéneo, que rechazó explícitamente las diferencias y atenuó las identidades locales y regionales, con miras a la formación de una ciudadanía disciplinada, al servicio de un proyecto que exalta la modernidad (Hobsbawm, 2013). Es esa escuela la que se ve hoy internacionalmente desafiada por la migración y por la recomposición de su tejido social, cultural y étnico.

El Estado nacional chileno y su escuela no constituyen una excepción a esta tendencia mundial. De allí que nuestro objetivo de investigación sea explicar la forma en que el personal docente percibe e interpreta el fenómeno de la inmigración que vive el país, así como las medidas que este mismo profesorado toma para abordar la niñez migrante en la escuela. Concretamente nos hemos preguntado: ¿Cómo percibe el personal docente esa nueva y compleja realidad? ¿Cómo la interpretan? ¿Qué estrategias adoptan para enfrentar la "otredad" representada por estudiantes con sus raíces en otro contexto cultural? 
http://doi.org/10.15359/ree.25-1.14

http://www.una.ac.cr/educare

educare@una.ac.cr

\section{Marco conceptual}

Uno de los supuestos teóricos que sustenta este estudio se expresa en la tesis siguiente: Los actores sociales construyen las estructuras de la sociedad y, a su vez, son estructurados por estas en un movimiento dinámico, dialéctico. Existe un diálogo entre estructuras-actores socialesacciones de los actores. Los actores sociales construyen la sociedad, pero, a su vez, son construidos por esta, aunque muchas veces las estructuras aparezcan en la conciencia de los sujetos como cosificadas, ajenas, alejadas de la praxis de los actores (Berger y Luckman, 2003; de la Garza Toledo, 2012; Lukács, 1990). En ese proceso de construcción de la sociedad, los actores interpretan el medio, atribuyen significados e intentan -a través de sus marcos interpretativos, en permanente interacción con otros sujetos- alcanzar comprensión de los fenómenos que tienen lugar en su entorno. Independientemente de la corrección o falsedad de sus interpretaciones, estas guían su accionar en la creación/construcción de las relaciones sociales (Gilje y Grimen, 2007).

Otra noción teórica fundamental de nuestra indagación consiste en ubicar las interpretaciones y lecturas de la realidad propuestas por los actores sociales, en relación con el contexto social en el cual se desenvuelven. La definición social de la realidad es contextual, adquiere significado en un determinado contexto, por ejemplo: o bien en el de una gran ciudad con características propias de la modernidad o bien en un contexto rural, tradicional (Berger y Luckman, 2003). Pero, limitarse exclusivamente a esta relación "contexto-definición social de la realidad" nos conduciría a esbozar una relación mecánica, que no sería dialéctica. Aparece lo fructífero y genuinamente dialéctico de esta relación cuando se considera en el análisis la "potencia realizadora" (Berger y Luckman, 2003, p. 219) de las lecturas de la realidad, de la definición social. Esta definición social de la realidad genera, a su vez, realidad y contribuye a formar identidades sociales en procesos que no están desprovistos de una carga emocional.

De estas nociones se desprende la necesidad de incluir en una indagación como esta las interpretaciones, las percepciones y las atribuciones de significados de los actores sociales; estamos ante una realidad que ya ha sido interpretada, en un verdadero esfuerzo hermenéutico, por los actores mismos; pero -y seguimos aquí las nociones epistemológicas/metodológicas de Giddens (2012)- limitarse solamente a este plano implica restringir la indagación a un nivel exclusivamente experiencial, extremadamente cercano a las nociones de la vida cotidiana, al sentido común'. Consideramos, desde esta perspectiva, que no basta solamente con repetir las interpretaciones de los actores sociales, sino que estas deben ser reconstruidas con el auxilio de conceptos teóricos, acuñados en el marco de las ciencias sociales, en el ejercicio de aquello que Giddens (2012, p. 207) ha denominado "hermenéutica doble". Esta práctica investigativa nos ha ayudado a transformar las descripciones de los actores sociales en categorías propias del discurso de la ciencia social.

\footnotetext{
${ }^{1}$ No desconocemos que las interpretaciones de las experiencias de los actores sociales en la vida cotidiana se efectúan con el auxilio de abstracciones (Pierce, 1990). Sin embargo, la tarea de cientistas sociales implica realizar interpretaciones de interpretaciones, esto es "interpretaciones de segundo grado" (Eco, 2000, p. 353).
}

Carlos Muñoz-Labraña, Luis Ajagán-Lester, Rosendo Martínez-Rodríguez, Bastián Torres-Durán, Carlos Muñoz-Grandón y Karen Gutiérrez-Cortés

Los artículos de la Revista Electrónica Educare del Centro de Investigación y Docencia en Educación de la Universidad Nacional, Costa Rica, se comparten bajo términos de la Licencia Creative Commons: Reconocimiento, No Comercial, Sin Obra Derivada 3.0 Costa Rica. Las autorizaciones adicionales a las aquí delimitadas se pueden obtener en el correo: educare@una.cr 


\section{Problema y objetivos}

El problema central de nuestra indagación consiste en capturar, describir y comprender las percepciones de los sujetos entrevistados en torno a la presencia de estudiantes migrantes en las escuelas municipalizadas. De manera que, cómo percibe el personal docente los desafíos que implican el arribo a la escuela municipalizada del Gran Concepción de estudiantes provenientes de otro contexto cultural, político y económico, constituye la pregunta fundamental de este estudio.

Los objetivos de la investigación son: a) Indagar sobre los procesos interpretativos que realiza el profesorado en torno al estudiantado migrante recientemente llegado a la escuela; b) Identificar las explicaciones que el personal docente crea/construye para dar cuenta de este fenómeno; c) Identificar las voces y acentos que cruzan y marcan el discurso del personal docente participante en este estudio; d) Comprender las valoraciones que los sujetos del estudio han acuñado en torno a la niñez migrante.

\section{Metodología}

Los métodos utilizados fueron de carácter cualitativo. El trabajo de campo fue realizado en cuatro establecimientos municipales del Gran Concepción, Chile. El criterio central de selección de estos establecimientos consistió en que hubiesen recibido estudiantes migrantes en los últimos dos años; los criterios de selección de los sujetos participantes en el estudio fueron estratégicos (Eneroth, 2009), se buscó la igualdad de género en la muestra de veinte docentes: diez mujeres y diez hombres, y que la totalidad hubiese tenido experiencias de aula con niños migrantes.

Los datos fueron recolectados durante el segundo semestre del 2017 y primer semestre del 2018, en forma de entrevistas profundas, semiestructuradas, previamente validadas por tres personas expertas. Las entrevistas tuvieron una duración promedio de dos horas, fueron grabadas y transcritas; el corpus está compuesto por una masa textual de 112456 palabras. Para el análisis utilizamos la técnica de la categorización de los textos recopilados, que implica una lectura cuidadosa y analítica de los diversos escritos para interpretar las potenciales categorías y los supuestos teóricos subyacentes (Kvale, 2011). Las categorías constituyen la dimensión fundamental de nuestro análisis; sin embargo, producto de la riqueza conceptual de algunas de ellas, fueron -siguiendo las recomendaciones metodológicas de Kvale (2011)-segmentadas en subcategorías. En ciertos casos, cuando algunos pasajes del material transcrito surgieron como especialmente complejos, utilizamos una mirada cuasi-naturalista (Ödman, 2007), que conlleva el ejercicio interpretativo de tomar distancia del material e iluminarlo con el auxilio de una bibliografía apropiada, lo que permitió la triangulación de la información. 
http://doi.org/10.15359/ree.25-1.14

http://www.una.ac.cr/educare

educare@una.ac.cr

\section{Resultados}

Del análisis del material empírico disponible, emergen las siguientes categorías:

1. La población migrante ha pasado a ser una preocupación en la Escuela. Esta es una de las cuestiones sobre las cuales existe consenso entre los sujetos entrevistados, quienes, a renglón seguido de dar cuenta de importantes preocupaciones de la escuela vinculadas especialmente a la violencia escolar, la evaluación de los aprendizajes y los resultados en el sistema de medición de la calidad de educación, señalan que, en los últimos años, ha emergido la preocupación por los grupos migrantes y su inserción en la vida escolar. Esto, debido principalmente al ingreso a la escuela de alumnado proveniente de otros países, quienes con su presencia han generado escenarios no previstos en su trayectoria profesional, ni en la de la escuela; lo que genera tensión en la institución escolar, situación que una parte del profesorado califica como de una sobrepoblación:

Esto es muy particular porque nosotros veíamos el tema de la migración muy lejana, pero desde hace tres años, de pronto, nos vimos con estudiantes de distintas nacionalidades y con una sobrepoblación, lo que nos ha cambiado la vida... Entonces algo que no era tema en la escuela ahora es un gran tema que nos tiene ocupados y preocupados, porque es una realidad que no conocíamos y hoy está en nuestra aula. (Pa3T2)

La experiencia de tener estudiantes de otras nacionalidades es vista con desconcierto por el profesorado $y$, al mismo tiempo, como un cambio trascendental en su realidad profesional. Reconocen que en el centro de esta nueva realidad hay niños y niñas, que no tienen responsabilidad de la complejidad de la nueva situación pedagógica:

Es como que de repente nos cambiaron las reglas del juego y ninguna autoridad hace nada, nadie dice nada, nadie explica nada y uno tiene que asumir no más porque al final del tema... los niños son los niños y ellos no tienen la culpa de esto que nos pasa a nosotros, de ser sorprendidos por la realidad, por eso yo pienso que hoy esto es una realidad muy cercana, para la cual tenemos que acostumbrarnos si o si... Como que no nos queda otra. (Po4T6)

Interesante resulta destacar la última frase "y ninguna autoridad hace nada, nadie dice nada, nadie explica nada...", manifestada por el personal docente. Su voz refleja y refracta, para usar la expresión de Volóshinov (2009), una visión de escuela y educación en la cual es la autoridad, en primer lugar, la llamada a enfrentar la problemática de la migración. Las estructuras sociales, hemos señalado, aparecen no pocas veces en la conciencia de los sujetos como cosificadas, ajenas, alejadas de su praxis. Es lo que testimonia este enunciado y es reafirmado por otros similares. El personal docente, inserto en un contexto en el que ejerce la hegemonía un paradigma tecno-burocrático, tiende a verse a sí mismo como ejecutor de medidas pensadas por otras instancias, más que como actores que pueden contribuir activamente a la construcción de una estructura social, en este caso la recepción de niños y niñas migrantes.

Carlos Muñoz-Labraña, Luis Ajagán-Lester, Rosendo Martínez-Rodríguez, Bastián Torres-Durán, Carlos Muñoz-Grandón y Karen Gutiérrez-Cortés

Los artículos de la Revista Electrónica Educare del Centro de Investigación y Docencia en Educación de la Universidad Nacional, Costa Rica, se comparten bajo términos de la Licencia Creative Commons: Reconocimiento, No Comercial, Sin Obra Derivada 3.0 Costa Rica. Las autorizaciones adicionales a las aquí delimitadas se pueden obtener en el correo: educare@una.cr 
http://doi.org/10.15359/ree.25-1.14

La última frase ilustra un cierto fatalismo, puesto que a su juicio no hay otra alternativa que "acostumbrarse" a esta nueva realidad, compleja, desconcertante, que debería ser modificada por la autoridad y no por el personal docente.

2. Un fenómeno que a ratos divide al profesorado. El aumento experimentado por la población migrante no solo genera tensión en docentes y en su relación con las autoridades de la escuela, sino que también entre los mismos grupos docentes, algunos de los cuales se resisten a enfrentar este nuevo desafío, dados los compromisos asumidos con antelación en los distintos instrumentos de gestión existentes, lo que, en su mayoría, presionan al profesorado por los logros de aprendizaje del estudiantado. Así, la incorporación de la nueva población escolar es vista con preocupación y cierta angustia por el profesorado:

Siempre han sido una preocupación nuestros niños y niñas. Lo que pasa que hoy tenemos una realidad que nos golpea y nos puso en discusión, porque hay colegas que como que se resisten a esa realidad y dicen, pero por qué nosotros, si tenemos tantos problemas para obtener buenos resultados y estamos tan presionados por eso... Imagínese las metas de lectura que tenemos y de comprensión lectora, ayudar a leer a un niño o niña que tiene un idioma que nosotros desconocemos, es muy complicado. Hoy tenemos que hacer frente a una realidad que no hemos pedido pero que está en la escuela, por el sector social que atendemos... Hay algunos que se resisten, pero otro en realidad dice que hay que tratar de hacer lo que se pueda y ahí estamos un poco divididos. (Pa1T5)

Los enunciados citados nos muestran la potencia realizadora del contexto, que contribuye a construir el marco interpretativo de los actores en su construcción de la realidad. Alcanzar buenos resultados aparece como una meta impuesta y de allí se desprende el sentir presión, lo que angustia al personal docente: estamos ante un contexto dominado por la racionalidad técnico eficientista, que privilegia la pedagogía por objetivos y resultados. A segundo plano pasa la problemática de seres humanos que han sido desgajados de su entorno, de sus hogares, de familiares que permanecen en el país de origen. En la interpretación de las personas entrevistadas, el rol docente parece reducirse a cumplir con las metas de lectura, a alcanzar una buena comprensión lectora, a "obtener buenos resultados."

3. Un proceso que parece no terminar. La división existente tiene un escenario inicial que fue visto como una novedad por el profesorado. Sin embargo, conforme avanza el año escolar y se leen las noticias de la prensa, el profesorado tiene la convicción que este escenario no es transitorio, sino que, de alguna manera, será un proceso largo que está lejos de concluir:

... al principio era curioso ver a niños de otras nacionalidades en la escuela y como que era novedad... Hoy ya no lo es, y pasó de novedad a una preocupación, por los desafíos y la responsabilidad que eso implica, porque el número de migrantes en el país sigue creciendo 
http://doi.org/10.15359/ree.25-1.14

http://www.una.ac.cr/educare

educare@una.ac.cr

vertiginosamente. Lo dicen a cada rato en las noticias..., y ya es con ver en Concepción caras de personas que no son chilenas sino extranjeras, junto a niños y niñas. Es un fenómeno que por el momento no parará de allí mi preocupación por lo que está pasando y vendrá, lo que de alguna manera me genera cierta tensión, porque los niños necesitarán un sistema educativo preparado para ellos y para enfrentar este desafío. (Pa1T8)

En este tipo de enunciados aparece una visión de la recepción del otro ser como un proceso alienado, ajeno a los actores. Es"el sistema educativo"el que debe responder a los desafíos que implica la persistencia de la migración. No hay indicios de actividades e iniciativas propias, de propuestas como el intercambio de experiencias en el trabajo con niños y niñas migrantes, de propuestas de lecturas sobre la temática, de la creación de talleres sobre cómo enfrentar esta nueva realidad.

La persistencia del fenómeno migratorio genera preocupación en el profesorado y cierta tensión e incertidumbre respecto de la pertinencia del sistema educativo en el futuro.

4. Un fenómeno para el cual el profesorado dice no estar preparado. La tensión advertida por el profesorado tiene como preocupación central su falta de preparación para enfrentar este desafío. Especialmente, porque se trata de un fenómeno para el cual la universidad no lo ha preparado; agrega, a renglón seguido, que tampoco las mismas universidades lo están, como prueba el hecho que ni siquiera estos centros de educación superior ofrecen cursos o actualizaciones en la materia:

La llegada de estos alumnos nos preocupa. Yo diría porque básicamente no tenemos herramientas para enfrentar es a realidad, a un a no la prepararon en la Universidad para esto, de hecho, es un tema nuevo para nosotros y para todo el mundo, incluidas las universidades que forman profesores, creo yo. Yo revisé si había cursos para hacer, pero no había nada, ni en la universidad ni en ninguna parte y por eso una como que se siente medio desamparada con lo que pasa. Imagínese, nuevamente nosotros los profesores debemos enfrentar nuevas realidades para las cuales no hemos sido formadas. (Pa3T4)

Las falencias en la formación inicial y la falta de cursos de actualización generan desazón no solo en el profesorado, sino también en los equipos de gestión de la escuela, quienes se sienten superados por la contingencia y hacen lo que pueden. De allí el llamado urgente de ayuda:

El problema es que la universidad no nos prepara para esto y eso se puede ver porque no existe ayuda de parte de nadie.... El rector y la jefe de UTP hacen lo que pueden... pero hace falta ayuda o sea personas que sepan cómo enfrentar esta cuestión. (Po7T11).

El personal entrevistado se ve inconscientemente como objeto de medidas de apoyo, más que como sujetos activos que pueden modificar la realidad, contribuyendo así a la cosificación de la educación. Nada indica, en los extractos analizados, que se sientan parte creadora de las estructuras educativas.

8

Carlos Muñoz-Labraña, Luis Ajagán-Lester, Rosendo Martínez-Rodríguez, Bastián Torres-Durán, Carlos Muñoz-Grandón y Karen Gutiérrez-Cortés

Los artículos de la Revista Electrónica Educare del Centro de Investigación y Docencia en Educación de la Universidad Nacional, Costa Rica, se comparten bajo términos de la Licencia Creative Commons: Reconocimiento, No Comercial, Sin Obra Derivada 3.0 Costa Rica. Las autorizaciones adicionales a las aquí delimitadas se pueden obtener en el correo: educare@una.cr 
5. Un proceso que obedece a la realidad que enfrentan otros países. Interesante resulta también destacar cómo el profesorado desarrolla un proceso hermenéutico al intentar comprender y explicar el fenómeno migratorio, apuntando a la situación en los países de origen de las personas migrantes. Así lo demuestra el siguiente relato que ve en el deterioro de la condición económica de nuestros países vecinos, la causal más importante del fenómeno migratorio, advirtiendo que, mientras ella no se resuelva, el proceso no se detendrá.

Si yo creo que mientras los otros países vecinos estén complicados económicamente este fenómeno continuará cada vez más. Yo veo las noticias más que antes, porque quisiera saber cómo va la cosa en los países desde donde vienen nuestros estudiantes para ver si este fenómeno se revertirá, y la respuesta siempre es la misma, mientras en los otros países esté la cosa como está, difícilmente este proceso migratorio se revertirá y hay que prepararse para ello. (Po2T6)

Hay quienes incluso van más allá y ofrecen como factor explicativo una condición económica favorable en nuestro país que, pese a tener un porcentaje de población vulnerable económicamente, genera atracción en el resto de los países vecinos. Lo que los hace demandar capacitación y perfeccionamiento para enfrentar su docencia, tal como lo demuestra el siguiente relato:

Definitivamente, el que nuestro país esté mejor que sus vecinos o un poco mejor diría yo, porque en nuestro país también hay pobreza y vulnerabilidad económica, y los diarios y la televisión parecen olvidarlo. Mientras eso se mantenga, o sea mientras nuestro país esté en mejor condición económica que sus vecinos, creo que nuestro país seguirá estando de moda y mientras esta situación no cambie en los demás países, creo que esto no va a cambiar. Yo creo que deberíamos acostumbrarnos, y trabajar, capacitarnos y perfeccionarnos para enfrentarlo. (Po8T6).

6. Un fenómeno que cuesta comprender y aceptar por parte del profesorado con más años de servicio. Este es quizás uno de los hallazgos más interesantes de esta investigación, dado que el profesorado reconoce explícitamente que al personal docente con más años de servicio le es más difícil aceptar el fenómeno de la migración. Situación que -de acuerdo con el mismo relato- es compartida por algunas personas apoderadas:

Esto cuesta especialmente a los profesores con más años de servicio que están más cansados y como que les cuesta más aceptar la realidad. Yo no quiero discriminar, pero lo que yo veo en mi escuela es que como que los jóvenes y los propios niños aceptan de mejor forma la diversidad en las aulas y se hacen amigo de los que llegan, mientras que los más adultos les cuesta más. De hecho, a los apoderados también les cuesta y hay algunos que no quieren que los niños migrantes tengan las mismas oportunidades que los niños chilenos. (Pa4T15) 
http://doi.org/10.15359/ree.25-1.14

http://www.una.ac.cr/educare

educare@una.ac.cr

Al preguntarles por qué las personas apoderadas piensan eso, las respuestas del personal docente no se dejan esperar. Levantan dos líneas argumentativas, que por su importancia hemos subcategorizado como de rechazo al otro ser.

En primer lugar, porque el personal docente entrevistado señala que las personas apoderadas no quieren que sus niños y niñas salgan perjudicados por atender a la niñez de otras nacionalidades, lo que, a juicio de las personas apoderadas, podría generar una desatención de sus hijos e hijas. Argumento que es respondido por el profesorado aludiendo a su profesionalismo, como se infiere del siguiente relato:

Yo he notado que la principal preocupación de algunos apoderados es que no quieren que, por atender a los niños de otras nacionalidades, se descuide a sus niños, quienes necesitan y requieren también una atención. Eso me lo han dicho ya varios apoderados, como que yo veo que hay un poco de egoísmo en eso porque en el fondo como que sienten que se les presta más atención a los otros niños y niñas, pero para mí todos son mis alumnos. Además, yo soy una profesional, eso lo tengo claro y se los digo a mis apoderados cada vez que sale el tema. (Pa9T22)

En segundo lugar, el personal docente aduce que las personas apoderadas niegan que la escuela posea como misión la educación de la niñez de las otras nacionalidades y abogan por privilegiar a los grupos connacionales. En su esfuerzo hermenéutico, los maestros y las maestras interpretan estas exclusiones como parte del egoísmo de las personas apoderadas, quienes incluso señalan que solo después de atender a la niñez connacional, y si quedan vacantes, se reciba una cantidad limitada de niñez extranjera, quienes, -de acuerdo con el mismo relato-, no deberían ser una preocupación del gobierno chileno, sino de los gobiernos de sus países de origen.

Esto resulta a veces muy complicado porque como que de repente a los apoderados les viene el egoísmo y me dicen que porqué debemos educar a niños migrantes, si primero deben ser los niños chilenos, y que la escuela debería preocuparse de tener bien a sus niños y si sobran vacantes recibir una cantidad limitada de extranjeros, porque el gobierno debe velar por eso y no por todos los niños del continente, que del resto se preocupen los otros gobiernos de los otros países. (Pa8T12).

El sujeto extranjero, extraño, ajeno, el otro ser -comentaba Bauman (1996), puede ser, o bien rechazado, excluido, vomitado del nuevo cuerpo (estrategia antropoémica), o puede ser aceptado, siempre y cuando se comporte y sea exactamente como nuestro grupo (estrategia antropofágica). Apreciamos en los extractos citados la estrategia antropoémica de la exclusión del otro ser: no merecen estudiar aquí, que su educación sea responsabilidad de sus países, son una amenaza para nuestros hijos e hijas, etc. 
7. Migrantes que complejizan el trabajo docente. Esta es otra perspectiva que podemos apreciar en los discursos del profesorado del estudio, que da cuenta del impacto que -a su juicio- posee la llegada de la infancia migrante dentro de sus salas de clases. La definen como compleja, adicional a las labores docentes que tradicionalmente poseen en el aula, dadas las carencias con las que llegan los niños y niñas migrantes:

Mi impresión es que de todas maneras nuestro trabajo docente se complica y se pone aún más complejo y cuesta más trabajar cuando tenemos niños de otras nacionalidades... si antes teníamos problemas con los chilenos hoy el tema es doblemente más complejo y no es que yo los discrimine..., solo estoy diciendo que es más complejo trabajar con niños y niñas de otras nacionalidades... porque sin niños que tienen muchas carencias y yo me doy cuenta de que eso genera muchas barreras. (Po7T10)

Al preguntar al profesorado acerca de a qué se refiere con las carencias con las cuales llegan estos niños y niñas, las respuestas no se dejan esperar.

- La presencia de experiencias distintas a la niñez chilena emerge como una categoría que en parte explica estas carencias. Con ella, el profesorado da cuenta de su desconocimiento de las experiencias escolares y socioeducativas de las personas recién llegadas, así como de los programas de estudio que fueron la base de su formación, variables que de acuerdo con los discursos de los maestros y las maestras complejizan su labor.

...ellos vienen de otros países con otras experiencias escolares y socioeducativas que una desconoce y a veces cuesta aprender y seguirlos de buenas a primera, y a veces una no sabe cómo enseñar y qué experiencias traen desde sus países de origen, entonces todo es más complicado. De verdad es más complicado porque cuesta imaginar esas experiencias, esos lugares, esas escuelas y esos maestros que han tenido la responsabilidad de su aprendizaje. Entonces, como digo, uno se complica al no tener antecedentes de los programas de estudio con los cuales ellos se formaron y todas esas cosas que son antecedentes importantes a la hora de enseñar. (Po5T9)

- La existencia de costumbres distintas también es relevada por el profesorado para explicar esta complejidad. Señalan que algunas son buenas, como un mejor uso del idioma y el respeto que dispensan a la profesora. Mientras que otras no lo son tanto, como el carácter reservado que en la mayoría de las veces expresan en el colegio y la falta de algunas normas de urbanidad.

La existencia de costumbres distintas es una variable para considerar. Algunas son buenas como un hablar más pausado y un mejor uso del idioma.... Otras no tanto. Son buenas por ejemplo el respeto que le tienen a uno y son malas de pronto en la forma en que resuelven sus 
http://doi.org/10.15359/ree.25-1.14

http://www.una.ac.cr/educare

educare@una.ac.cr

conflictos y en la forma en que se relacionan con el resto de sus compañeros, porque son muy retraídos, no hablan mucho y como que uno no sabe quéestán pensando. Tampoco son buenas algunas normas básicas de urbanidad. Entonces, eso es como difícil para una manejar. Porque esas costumbres son distintas a las que tenemos acá y eso se nota. Yo no lo sé explicar muy bien, pero creo que tiene que ver con la idiosincrasia que tienen o algo así. (Pa12T13)

- La falta de conocimientos previos para enfrentar las materias complejiza también la labor docente, especialmente porque el profesorado tiene conciencia de ciertos vacíos curriculares que son claves a la hora de aprender nuevos contenidos que la niñez migrante no posee, no porque no quieran, sino porque la mayoría de las veces no han sido objeto de estudio en el sistema escolar de origen. Esto produce tensión a la hora de pretender promover nuevos aprendizajes.

...algunos vienen muy atrasados y traen vacíos que son fuertes. Aunque hay algunas excepciones. Pero en general yo noto que vienen con un vacío en sus colegios de procedencia, y yo he detectado que el currículo de origen simplemente no contemplaba ciertas materias. De hecho, me dicen que no siempre les enseñaban en el colegio y como que el sistema educativo no estaba tan organizado y era más desorganizado que acá en Chile. Por eso a ellos les cuesta más y a uno le resulta más difícil enseñar. (Po1T35)

- La existencia de carencias económicas. El profesorado quiere dejar testimonio de la condición de extrema vulnerabilidad con la que arriba la niñez recién llegada, señalando que, en el contexto chileno, estos grupos son vulnerables dentro de los niños y niñas vulnerables del país y, por tanto, esta condición complejiza aún más su labor. Reconocen ya tener un gran desafío para que la niñez que atienden regularmente pueda aprender en su contexto de vulnerabilidad, y preparar clases para quienes se encuentran en extrema vulnerabilidad es aún más complejo.

...eso se ve inmediatamente porque en el fondo tenemos estudiantes vulnerables a los cuales resulta difícil enseñar, pero los niños migrantes son aún más vulnerables que ellos, tienen condiciones económicas muy difíciles que cuesta hacer para que superen. De hecho, ese quizás es un gran problema que atraviesa todo lo que una observa. Ahora es claro que les faltan recursos y la escuela algo trata de ayudar, pero ellos cuentan su precariedad. Imagínense, viven de allegados muchas personas, mezclados entre adultos y niños... De tal manera que para ellos es difícil su inserción, no solo en la escuela, sino que también en el país. (Pa712T9)

No es que no tengamos nosotros niños vulnerables. Lo que quiero decir es que estos niños son más vulnerables que los que tenemos acá, y eso es lamentable. (Po9T25) 
Por anteriores investigaciones (Muñoz, Ajagan, Sáez, Cea y Luengo, 2013), sabemos que los niños y niñas que no poseen un capital cultural reconocido por la escuela encuentran serias dificultades en su encuentro con la educación formal. Este fenómeno se agudiza notablemente en el caso de niños y niñas aún más vulnerables que los nacionales, como lo es la población migrante de escasos recursos.

8. Valoraciones positivas en la nueva realidad que construyen los grupos migrantes. A pesar del listado de situaciones complejas, que -de acuerdo con las percepciones del profesorado en estudio- genera la presencia de una población migrante en las aulas, el mismo profesorado reconoce la existencia de algunos interesantes efectos positivos a destacar. Lo que hace que el profesorado justiprecie el conjunto de observaciones realizadas con anterioridad sobre el fenómeno en estudio.

- Un proceso que ha generado un aumento en la matrícula. La llegada de migrantes ha generado un aumento de matrícula en la escuela. Lo que es valorado por el profesorado, especialmente dada la realidad educacional de nuestro país, que en los últimos años ha asistido a una pérdida de matrícula sistemática del sistema municipal, en beneficio de la educación particular subvencionada. De allí que la llegada de la población migrante es vista como un hecho positivo, dado que esta viene a paliar en parte la notoria baja de matrícula existente. Veamos los siguientes relatos.

Ahora, no hay que desconocer que venía bajando su matrícula producto que las escuelas particulares subvencionadas se han llevado a nuestros mejores alumnos y ahora tenemos cada vez más estudiantes, pero de otras nacionalidades en forma creciente y por eso este proceso yo veo que no termina y a lo mejor pasarán años y no terminará. (Pa1T8)

Hasta lo que yo veo, no tenemos más que tratar de hacer las cosas bien porque cada vez tenemos más alumnos de otras nacionalidades. La matrícula ha aumentado gracias a eso, y eso es positivo porque aumenta la posibilidad de dar continuidad a la escuela y alejar el fantasma de despoblación estudiantil que afecta a algunas escuelas municipales. (PO9T9)

Del último relato se desprende que el aumento de matrícula no solo es un índice numérico para exhibir, sino que también constituye una forma que asegura la fuente laboral del profesorado en el futuro, liberando al personal docente de las preocupaciones que viven otras escuelas de la misma dependencia.

- Una forma de llevar a las aulas la diversidad. Otra dimensión positiva que surge de los relatos está asociada a la existencia de un fenómeno nuevo que emerge en las aulas de clase, que hace que, tanto el profesorado como los niños y niñas puedan acceder a relatos, paisajes, realidades y culturas diversas que, de no mediar la presencia de las niñas y niños migrantes, sería muy difícil vivenciar. Esto, porque el profesorado tiene la convicción de que estamos en presencia de un fenómeno que va más allá de la 
http://doi.org/10.15359/ree.25-1.14

http://www.una.ac.cr/educare

educare@una.ac.cr

presencia de los niños y niñas en sus aulas, quienes son portadores de una herencia cultural familiar y social que contribuye a valorar la existencia de una pluralidad sociocultural que actúa en doble sentido. Primero, porque contribuye a reconocer la existencia de otros seres desde la cotidianeidad. Segundo, porque este ejercicio conlleva la posibilidad de mirarse, a sí mismo o a sí misma.

Tener estudiantes de otras nacionalidades también trae algunos beneficios, especialmente al momento de no solo hablar de la diversidad, sino que también hacerla visible en las clases, experiencia que nuestros niños y niñas no habrían podido tener jamás, dada su condición de vulnerabilidad y, por tanto, acceso a relacionarse con personas de otras nacionalidades y culturas, aunque hablen el mismo idioma siempre es una contribución. Los niños migrantes yo diría que aportan eso, miradas distintas. El saber de los otros, en las formas de ver la vida y la realidad o valorar algunos aspectos de la vida que a veces uno $\mathrm{ni}$ siquiera valora ni menos los niños, porque como que una se acostumbra a cierta comodidad y paisajes... Ellos describen sus hogares y lo hacen como todos los niños con una inocencia que conmueve a veces porque como que una se estremece al escuchar algunos relatos de carencias fuertes, desamparo del Estado, lo que es ratificado por sus madres. (Pa8T20)

- Concienciación de una realidad dolorosa. Al conocerla genera empatía dentro de la comunidad escolar. Algunos de los relatos de los niños y niñas migrantes dan cuenta de un conjunto de experiencias dolorosas a muy temprana edad, que hacen que la comunidad escolar no solo tome mayor conciencia de la realidad que enfrenta el migrante y de las razones que llevaron a sus padres a tomar tal decisión, sino que también, contribuye a tomar mayor conciencia de la dolorosa realidad que también vive la niñez en nuestro país, quienes, además de tener una condición de vulnerabilidad no elegida, sufren la vulneración de sus más elementales derechos, como tener acceso a una vivienda y a una salud adecuada.

Yo he escuchado de todo, los niños dicen y hablan cosas con sentimiento y una gran ingenuidad y no solo aportan ingenuidad, sino que expresan humanidad que uno no puede permanecer como si nada frente a tanta injusticia y carencias. A veces pienso cómo puede ser esto... y pienso que es una realidad aumentada a la que viven muchos de nuestros niños, quienes también sufren vulneración de derechos y como que a propósito de los niños que llegan... uno tiene mayor conciencia y empatía con la realidad de nuestros propios niños que no tiene una vivienda o acceso a salud, como la realidad que enfrentan los que llegan de otros países, quienes lo pasan peor. (Po3T22)

Claro, el mundo es diverso en teoría, hasta que a uno le toca vivir una realidad más allá de las aulas es que toma real conciencia. No es que no esté consciente de las carencias de mis alumnos y alumnas y de la diversidad que existe en nuestro país, lo que pasa es que 
es otra como diversidad, se entremezclan carencias económicas con carencias afectivas, y la más importante, la imposibilidad de vivir en su propio país con su propia gente, con sus espacios que vieron desde niños, sus costumbres, comidas, familiares, casas... por muy precarias que estas sean. Por lo que hablamos de una diversidad que reúne un conjunto de carencias que estos estudiantes migrantes deben enfrentar desde muy chiquititos y yo veo cómo ellos tratan de asimilar y me pongo en el caso de ellos, es muy complejo lo que enfrentan. (Po5T28)

- Justipreciar la realidad del país. Los relatos de los niños y niñas migrantes generan en el profesorado muchas reflexiones y cuestionamientos respecto al sentido que tiene la migración y, sobre todo, la incidencia de la vulnerabilidad en este proceso. Es interesante que el profesorado termine por valorar positivamente la realidad del país, pese a la precariedad con que gran cantidad de sus compatriotas enfrentan el día a día, lo que hace que tomen conciencia de cómo la niñez migrante y nacional debe hacer frente a esa realidad, enfrentando además sus procesos formativos.

Una como que no se da cuenta que, en América Latina, hay gente que lo pasa muy mal, y algunas de ellas están en Chile hoy. Entonces como que para mí los migrantes ponen una cuota de realidad de lo que tenemos como país. Es verdad que nos falta mucho, pero tenemos mucho más que muchos otros países, cuyos habitantes no tienen lo mínimo para vivir y deben migrar. (Pa4T3)

Como profesora esto a mí me ha ayudado y creo que a algunos alumnos también. Nos ha ayudado a valorar lo que tenemos aún con sus problemas apremiantes, sus injusticias y falta de solidaridad con los más pobres, los mapuches o las minorías sexuales. Chile es un país desigual, pero aún con esta desigualdad es mejor que la realidad que enfrentan estos alumnos y alumnas en sus países de origen. Razón por la cual he aprendido a valorar más lo que tenemos. Y creo que algunos de mis alumnos y apoderados también. (Pa10T3)

- Valorar la importancia del lenguaje para comunicarse. El profesorado reconoce que la llegada de la población migrante genera una revalorización de la comunicación y del lenguaje como medio para que ello se produzca. No es que no estén conscientes de su importancia, sino que, a partir de la experiencia que están viviendo, son capaces de apreciar mucho más su función, proporcionando una valiosa oportunidad como medio que facilita el contacto y el aprendizaje entre los seres humanos. Son capaces de advertir matices idiomáticos y confrontarlos con lo que ocurre a nivel nacional:

No cabe duda de que otra cuestión que uno valora es la importancia del lenguaje para comunicarse, el español, esto porque salvo los niños haitianos el resto todos hablan nuestro mismo idioma. Como que las fronteras de comunicación se ensanchan y tanto nosotros, los profesores como los alumnos se pueden comunicar, y eso es a lo mejor una cosa obvia, 
http://doi.org/10.15359/ree.25-1.14

http://www.una.ac.cr/educare

educare@una.ac.cr

pero no es tan obvia hasta que los vives, como nosotros lo estamos viviendo. Una sabe que en Perú, Colombia, Ecuador, se habla español, pero en estos casos el idioma adquiere otra dimensión, porque es como el elemento común, el cemento que está a la base de todo lo que podemos hacer y nos facilita la comunicación y el aprendizaje. Por lo que es más complejo con los niños haitianos porque ellos no hablan español en su casa y les cuesta más. Ahora que, además, una se da cuenta que como que nosotros no hablamos muy bien el idioma y da gusto escuchar a niños peruanos y colombianos hablar. (Pa2T15)

- Respeto con el profesorado. Finalmente, una última característica positiva valorada por el profesorado es el respeto que les tienen las niñas y niñas migrantes. Condición que también advierten en sus padres, madres o personas apoderadas, quienes se relacionan en un marco de respeto adecuado con el profesorado, lo que tributa directamente no solo en la autoestima del profesorado, sino también en su condición de profesional de la educación:

Esta es una cualidad que yo he notado que es como diferente. Hay una valoración de la figura del profesor que se nota en los niños y en los padres, madres y apoderados en general. Cuando los niños se refieren a uno lo hacen con mucho respecto, siempre de manera respetuosa, pausada, como comunicándose con una persona que ellos sienten es una autoridad en el colegio y en la sociedad, y eso me gusta y yo les digo al resto de los niños que eso es un ejemplo de cómo se debe tratar al profesor, $y$, de paso, uno se siente como un verdadero profesional de la educación. (Po7T30)

\section{Interpretando las interpretaciones}

Hemos presentado, hasta aquí, la categorización del material empírico, buscando rescatar las percepciones e interpretaciones del personal docente sujeto de nuestro estudio. En un movimiento interpretativo en espiral, presentamos a continuación la reinterpretación del material, en un esfuerzo hermenéutico, apoyándonos en conceptos teóricos adecuados a la temática de la escuela y la migración.

La lectura de las categorías y subcategorías presentadas nos hace sentir los ecos de una profunda contradicción entre la racionalidad instrumental, eficientista del Estado nacional por una parte $y$, por otra, una sociedad que sufre una transformación vertiginosa y que tiende hoy a ser pluricultural; la escuela del Estado nacional ha negado la diversidad, la ha expulsado de la normalidad que pretende crear para construir una ciudadanía culturalmente homogénea, disciplinada, dispuesta incluso a dar la vida por la nación (Anderson, 2006; Hobsbawm, 2013). La presencia de niñez migrante implica una ruptura con la normalidad vulnerable de las escuelas municipalizadas, pero percibida e interpretada por el personal docente como la conocida vulnerabilidad familiar. A la habitual, se suma una nueva vulnerabilidad, la de los Otros seres, la de 
http://doi.org/10.15359/ree.25-1.14

personas extranjeras pobres que, incluso -en algunos casos-, hablan otra lengua, heteroglosia ${ }^{2}$ que agrega nuevos obstáculos a la docencia.

Del material y de las categorías surgen voces entre el personal docente que dan cuenta de la presión permanente ejercida por el Ministerio Nacional de Educación (MINEDUC), percibido como una estructura ajena a la praxis del profesorado; la aplastante exigencia de alcanzar buenos resultados se convierte en aún más aplastante en este nuevo contexto. Ese nuevo contexto ofrece una realidad cambiante, compleja, dinámica. Por una parte, hay padres y madres - de acuerdo con el relato del personal docente-que pretenden expulsar del espacio social nuestro al estudiantado extranjero; en esa lectura la niñez migrante es percibida como distinta a nosotros, como amenaza y sin los mismos derechos que la niñez nacional. Por otra, se pueden notar acentos en los discursos, para usar el término de Volóshinov (2009), que valoran profundamente el aporte que la presencia de estos Otros seres conlleva, puesto que permiten entender la realidad y las asignaturas desde otra perspectiva, enriquecedora. Y, en una compleja espiral de interpretaciones, surgen otras voces que destacan las carencias de los Otros seres y enfatizan su propio desconcierto, angustia y preocupación.

Desde una perspectiva semiótica (Ajagan Lester y Muñoz Labraña, 2017) podemos señalar que, cómo los actores sociales construyan el espacio social nuestro - de nuestra cultura, la normal, la correcta, la legítima-, es fundamental para la creación/construcción de identidades sociales. Ese espacio social no constituye una estructura estática, sino que se trata de un proceso en el que se perciben conflictos e interpretaciones distintas, en este caso sobre la migración. Podemos apreciar, por un lado, a quienes aceptan a los grupos migrantes y, por otro, quienes los rechazan o consideran que no pueden trabajar con ellos. Si se impone la aceptación de migrantes, se abre la posibilidad del reconocimiento como personas tan válidas como cualquier estudiante nacional (Taylor, 1994); si se impone el rechazo, pasarán a ser los ajenos, el estudiantado que no pertenece a nuestra comunidad, el estudiantado ellos, el estudiantado excluido (Álund, 1997).

La clásica distinción de la semiótica cultural (Lotman, 1990) de límites entre el espacio de ellos/ellas (los seres otros, los grupos ajenos, los individuos hostiles) y nosotros/nosotras (los grupos nuestros, cultivados, normales) nos puede hacer comprender que el arribo de estudiantes del extranjero implica problemas técnicos e identitarios, en tanto su presencia cuestiona nuestra propia identidad nacional, la chilena. Por ejemplo: ¿Hasta qué punto los relatos chauvinistas sobre la Guerra del Salitre o del Pacífico siguen siendo legítimos y válidos cuando en nuestras aulas hay estudiantes de origen boliviano y peruano?, ¿puede una escuela que ha sido degradada -como señala Touraine (1997) al referirse a las escuelas públicas de Occidente-crear una comunicación intercultural?, ¿qué tipo de relatos requiere una escuela pluricultural?

${ }^{2}$ El término heteroglosia designa en este contexto: diferentes lenguas nacionales en el interior de la misma cultura (Vice, 1997). 
http://doi.org/10.15359/ree.25-1.14

http://www.una.ac.cr/educare

educare@una.ac.cr

\section{Comentarios y conclusiones}

No cabe duda de que la migración masiva ha sido un fenómeno nuevo en el país, que ha puesto a prueba la capacidad de nuestra sociedad para sintonizar con demandas de pueblos vecinos, tal como en el pasado lo hicieron y en el presente lo siguen haciendo nuestros connacionales, ya sea por dolorosas situaciones internas, o por el solo hecho de querer buscar otras oportunidades de vida.

Con el propósito de ilustrar mejor lo manifestado por el personal docente estudiado, resulta interesante incorporar algunos datos de contexto. El Instituto Nacional de Estadísticas (INE) y Dirección por la Comunidad de Chilenos en el Exterior (DICOEX) (2017) recientemente reveló, por ejemplo, que 1037346 chilenos y chilenas viven en el exterior, y que el 40,2\% lo hace en Argentina, lo que representa un aumento de 20,94\% en los últimos 12 años; es decir, 150 mil personas más en comparación a la última medición realizada en 2005. Del resto, un 13,4\% lo hace en Estados Unidos y el 10,2\% en España. El 87,2\% de personas chilenas que vive en el extranjero lo hace por un ingreso. De esta cifra, un 65,9\% trabaja de forma dependiente, el 22,7\% lo hace por cuenta propia (independiente) y el 6,4\% figura como empleador o empleadora (INE y DICOEX, 2017).

Las cifras globales entregadas por el INE son interesantes de confrontar con otras entregadas recientemente por el Gobierno de Chile (Instituto Nacional de Estadísticas [INE] y Departamento de Extrajería y Migración [DEM], 2019), según las cuales, el número de personas extranjeras en el país asciende a 1251225 al 31 de diciembre de 2018. Lo que representa cerca de un $7 \%$ de la población total del país. Del total, un 72\% reside en dos regiones: Metropolitana (62\%) y Antofagasta (10\%). Mientras que $28 \%$ son de nacionalidad peruana, un $14 \%$ es de origen colombiano, un $14 \%$ venezolano, un $12 \%$ boliviano y un $11 \%$ haitiano.

Al confrontar estos datos se puede concluir que la población migrante residente en Chile es bastante similar a la población chilena en el extranjero. De tal manera que estaríamos lejos de una sobrepoblación, como ha señalado parte del personal docente entrevistado.

Si se mira el conjunto del sistema educativo, integrado por tres subsistemas marcadamente diferenciados (municipal, particular subvencionado y particular), la llegada de niñez migrante no constituye un desafío para el conjunto del sistema, sino que solo para el sistema municipal, dada la situación socioeconómica de quienes han inmigrado recientemente.

A la luz de los antecedentes presentados sobre la migración en nuestro país, así como de los resultados obtenidos en la investigación, es evidente que el fenómeno migratorio tensiona al profesorado, especialmente para quienes tienen más años de docencia. Esto porque es un evento del cual se tiene conciencia que llegó para quedarse, sin precedentes en la historia educacional de nuestro país, y para el cual no han recibido preparación. Sienten que no poseen 
http://doi.org/10.15359/ree.25-1.14

las herramientas mínimas para enfrentar con éxito el desafío de atender en un mismo espacio a estudiantes de cuatro nacionalidades distintas, con experiencias de vida y herencias culturales disímiles, aunque compartan un idioma.

Las aprehensiones del profesorado son compartidas por algunas madres, padres y personas apoderadas, quienes no trepidan en defender erróneamente a sus pupilos y pupilas ante una supuesta mayor atención hacia quienes han llegado recientemente. Trazado existencial de límites que expulsa del nosotros a la población migrante y les percibe como amenaza.

El conjunto de tensiones y aprehensiones descritas evidencian un legítimo sentimiento de preocupación de parte del profesorado, quienes ven cómo sus aulas homogeneizadas por décadas por factores esencialmente socioeconómicos hoy reciben una población migrante con herencias culturales y experiencias de vida distintas. Esto demuestra, por un lado, una clara conciencia del rol que les corresponde como gestores de aprendizaje de sus estudiantes y, por otro, la necesidad de una política pública que les acompañe en este esfuerzo. De hecho, al analizar detenidamente las tensiones y aprehensiones del profesorado, emerge con fuerza la necesidad de recibir ayuda en este cometido, para el cual señalan que no se sienten preparados, abriendo paso, a través de este expediente, a la solución y a un abanico de fortalezas y potencialidades que el sistema educativo puede capitalizar sin mayor cuestión.

Lo anterior nos hace preguntarnos: ¿Qué pasaría si el Estado se hace parte de la solución y entrega los recursos adecuados para ayudar al profesorado en esta misión? ¿Cómo enfrentar el desafío de esta nueva escuela que se va transformando en pluricultural? ¿Basta con crear y conocer nuevas técnicas de enseñanza para esos seres humanos distintos a nuestra normalidad? ¿Es solo un problema técnico o se trata también de la aceptación del "Otro ser"y de su reconocimiento? El reconocimiento, para que sea tal, debe ser recíproco (Elster, 1986). De las categorías propuestas por nuestro análisis surge que tanto estudiantes como padres y madres extranjeras muestran respeto por el personal docente; de la polifonía discursiva que se escucha en los textos de las entrevistas podemos ver indicios de que este proceso, por hoy, no parece ser del todo recíproco.

Hasta ahora el profesorado se ha dado cuenta de que la inmigración genera un aumento de matrícula y que quienes llegan fortalecen un conjunto de principios y valores que podrían fortificar su labor. Ello genera un círculo virtuoso fundamental para enseñar a todos los niños y las niñas de la escuela el valor de la solidaridad, la integración, la diversidad, el respeto y la inclusión. No porque lo señalen los planes o programas de estudio, el libro de texto o las leyes de convivencia escolar, de formación ciudadana o inclusión, sino porque se educa en un espacio que hace realidad y vivencia un aprendizaje libre de discriminación. 
http://doi.org/10.15359/ree.25-1.14

http://www.una.ac.cr/educare

educare@una.ac.cr

\section{Declaración de Material complementario}

Este artículo tiene disponible, como material complementario:

-La versión preprint del artículo en https://doi.org/10.5281/zenodo.3661283

\section{Referencias}

Ajagan Lester, L. (2016). Det interkulturella mötet och hermeneutiken i globaliseringens tid. En F.M. Kirsch, R. León Rosales y C. Rodell Olgaç (Reds.), Vem vågar vara interkulturell? En vänbok till Pirjo Lahdenperä (pp. 147-158). TRT.

Ajagan Lester, L. y Muñoz Labraña, C. (2017). Siete tesis en torno al texto escolar como artefacto cultural. Revista Brasileira de Educação, 23, 1-19. https://dx.doi.org/10.1590/s141324782018230070

Álund, A. (1997). Multikultiungdom. Kön, etnicitet, identitet. Studentlitteratur.

Anderson, B. (2006). Comunidades imaginadas. Reflexiones sobre el origen y la difusión del nacionalismo. Fondo de Cultura Económica.

Bauman, Z. (1996). Postmodern etik. Bokförlaget Daidalos.

Berger, P. L. y Luckmann, T. (2003). La construcción social de la realidad. Amorrortu Editores.

de la Garza Toledo, E. (2012). La metodología marxista y el configuracionismo latinoamericano. En E. de la Garza Toledo y G. Leyva (Eds.), Tratado de metodología de las ciencias sociales: Perspectivas actuales (pp. 229-255). Fondo de Cultura Económica.

Eco, U. (2000). De los espejos y otros ensayos. Lumen.

Elster, J. (1986). Vetenskapliga förklaringar. Korpen.

Eneroth, B. (2009). Kvalitativ metod för samhällsvetenskaplig forskning. Akademilitteratur.

Giddens, A. (2012). Las nuevas reglas del método sociológico. Crítica positiva de las sociologías comprensivas. Amorrortu Editores.

Gilje, N. y Grimen, H. (2007). Samhällsvetenskapernas förutsättningar. Daidalos.

Hobsbawm, E. J. (2013). Naciones y nacionalismo desde 1780. Crítica.

Instituto Nacional de Estadísticas y Departamento de Extrajería y Migración y Departamento de Extrajería y Migración [DEM]. (2019). Estimación de personas extranjeras residentes en Chile al 31 de diciembre de 2018. https://www.extranjeria.gob.cl/media/2019/07/ Estimaci\%C3\%B3n-Poblaci\%C3\%B3n-Extranjera-en-Chile.pdf 
http://doi.org/10.15359/ree.25-1.14

http://www.una.ac.cr/educare educare@una.ac.cr

Instituto Nacional de Estadísticas y Departamento de Extrajería y Migración y Dirección para la Comunidad de Chilenos en el Exterior. (2017). Segundo registro de chilenos en el exterior ¿Cuántos son, dónde están y cómo son los chilenos? https://chilesomostodos.gob.cl/ chilesomostodos/documentos/segundo-registro-de-chilenos-en-el-exterior-dicoex-ine

Kvale, S. (2011). Las entrevistas en investigación cualitativa. Morata.

Lotman, Y. M. (1990). Universe of the mind. A semiotic theory of culture. Tauris Publishers.

Lukács, G. (1990). History and class consciousness. Studies in marxist dialectics. Merlin Press.

Ödman, P.-J. (2007). Tolkning, förståelse vetande: Hermeneutik i teori och praktik. Nordstedts Akademiska Förlag.

Pierce, C. S. (1990). Pragmatism och kosmologi. Daidalos.

Taylor, C. (1994). Det mångkulturella samhället och erkännandets politik. Daidalos.

Touraine, A. (1997). ¿Podremos vivir todos juntos? La discusión pendiente: El destino del hombre en la aldea global. Fondo de Cultura Económica.

Vice, S. (1997). Introducing Bakhtin. Manchester University Press.

Volóshinov, V. N. (2009). El marxismo y la filosofía del lenguaje. Los principales problemas del método sociológico en la ciencia del lenguaje. Ediciones Godot. 